Original Research Article

\title{
Genetic variation of CYP3A and its influence on the pharmacokinetics of tacrolimus, a calcineurin inhibitor
}

\author{
Ajay Kumar Gupta ${ }^{1}$, M. M. Ramashankar ${ }^{2}$, Mahadevan Kumar ${ }^{3 *}$
}

\begin{abstract}
${ }^{1}$ Department of Pharmacology,
${ }^{2}$ Department of Surgery, Army

College of Medical Sciences,

New Delhi, India

${ }^{3}$ Department of Lab Sciences

and Molecular Medicine, Army

Hospital Research and Referral,

New Delhi, India

Received: 21 May 2019

Revised: 02 August 2019

Accepted: 08 August 2019

*Correspondence to:

Dr. Mahadevan Kumar,

Email: dr.mkumar011

@gmail.com

Copyright: (C) the author(s), publisher and licensee Medip Academy. This is an openaccess article distributed under the terms of the Creative Commons Attribution NonCommercial License, which permits unrestricted noncommercial use, distribution, and reproduction in any medium, provided the original work is properly cited.
\end{abstract}

\begin{abstract}
Background: Tacrolimus (Tac) a calcineurin inhibitor (CNI), is a potent immunosuppressive drug which is widely used in organ transplant recipients. The drug has a narrow therapeutic window and high inter-individual pharmacokinetic variability. Tac is metabolized by cytochrome P450 3A (CYP3A) enzymes. The CYP3A5 activity is largely determined by the single nucleotide variant (SNV) CYP3A5*3 (c.219-237A>G; rs776746), which results in alternate mRNA splicing and a non-functional protein.

Methods: An observational prospective study was carried out at nephrology centre of army hospital where the consenting patients were enrolled in the study. The whole blood sample drawn was utilized to analyze for plasma drug concentration of Tac and genotyping for the CYP3A*5 polymorphism by the method as described by Cheung et al.

Results: 100 patients participated with an average of $98 \pm 8$ days after transplantation. Recipients having heterozygous CYP3A5*1 genotype manifested $70 \%$ lower mean steady state concentration of Tac $\left(\mathrm{C}_{0} / \mathrm{D}\right.$ ratio $)$ $1.67 \pm 0.43 \mu \mathrm{g} / \mathrm{l} / \mathrm{mg}$ in comparison to homozygote carriers of CYP3A5*3 $5.60 \pm 1.94 \mu \mathrm{g} / \mathrm{l} / \mathrm{mg} \mathrm{p}<0.001$.

Conclusions: Study suggested that Indian sub population closely mimics Caucasian population in terms of genotype expression for CYP3A5*3, who are expressing a non-functional enzyme for metabolism. Also, this study reinforces that population genotype CYP $3 A 5^{*} 1$ is significantly associated with lower $\mathrm{C}_{0} / \mathrm{D}$ ratio for Tac than the ones expressing homozygous CYP3A $5 * 3$ genotype. It also established the importance of pre-transplant genotyping, for better individualization of Tac doses. Further studies are suggested for population pharmacokinetic modelling study in future to derive starting dosage of Tac based on profiling of CYP3 genetics in recipients.
\end{abstract}

Keywords: Pharmacogenetics, Tacrolimus, Calcineurin inhibitor, Renal transplant

\section{INTRODUCTION}

Tacrolimus (Tac), a calcineurin inhibitor (CNI) of fungal origin is a macrolide lactone which has strong immunosuppressive properties. It's immunosuppressive efficacy is explained by its predominant actions of inhibition to mixed lymphocyte reactivity and the stimulation of cytotoxic T cells. It's additional actions are also known by suppressing cytokines production like IL2 as well as suppressing the expression of IL-2 receptors on activated T cells. ${ }^{1,2}$ It was first of all approved in the United States in 1994 where after, it has been cornerstone for the maintenance treatment protocol in many solid organs transplants like kidney, heart, and bone marrow. ${ }^{3}$ The drug has a very narrow therapeutic index and a relatively high pharmacokinetic variance which often leads to undesirable clinical outcome. ${ }^{4}$ At one end overexposure of the Tac risks serious toxicities and thus leads to poor tolerability which impacts adversely the long-term allograft and patient survival, whereas suboptimal therapeutic plasma concentration threatens precious transplant rejection and thus endangers the life of the person. ${ }^{5}$ Also, Tac is known to have considerable intra and inter individual pharmacokinetic (PK) variability, which leads to a poor correlation between dose and blood concentration and thus is a nightmare for 
clinicians many times requiring to put patients for frequent therapeutic drug monitoring (TDM). ${ }^{4,6}$

Various studies have identified multiple factors for pharmacokinetic variability like- type of transplant organ, age, race, status of hepatitis $\mathrm{C}$, diabetes, diurnal rhythm, food, liver function, haematocrit, serum albumin, cytochrome $\mathrm{P} 450$ isoenzyme, $\mathrm{P}$-glycoprotein genotype and phenotype, diarrhea etc. ${ }^{7}$ Thus, in past few years, various attempts have been made to establish the key factors amongst many factors known to be responsible for variation of plasma drug levels of Tac. ${ }^{8}$ One of the most important factor till date identified are genetic factors of the recipient like the genes coding for metabolizing enzymes in the liver and drug transporters in cell membrane. These genes are now understood to be most important factor contributing to pharmacokinetic variations of the drug plasma level. ${ }^{9}$ Tacunlike most other drugs, is bound to intracellular specific binding proteins within erythrocytes. As the drug is released slowly from these erythrocytes, it undergoes extensive metabolism by hepatocytes. The drug is predominantly eliminated through biliary route, mainly as inactive drug metabolites while less than $5 \%$ excreted in urine. ${ }^{10}$

Tacrolimus is primarily metabolized by subfamily of the cytochrome P450 (CYP) enzymes-like CYP3A4, CYP3A5, CYP3A7, and CYP3A43 which are the most versatile bio-transformation systems. ${ }^{11}$ Together, CYP3A4 and CYP3A5 account for almost $30 \%$ of hepatic cytochrome $\mathrm{P} 450$ activity. ${ }^{11} \mathrm{CYP} 3 \mathrm{~A}$ is existing in two isomeric forms which are responsible for Tac metabolism.CYP3A4 enzyme isoforms are almost expressed in all human beings' in liver and small intestine. Those individuals who are carriers of allele CYP3A5*1 polymorphically express CYP3A5 in tissues like liver, small intestine, kidney and some other organs. ${ }^{12}$ The gene for CYP3A5 enzyme is located on chromosome $7 \mathrm{q} 22.1$ on the minus chromosomal strand which consists of nine exons which encodes a 502amino-acid protein. ${ }^{11}$

Polymorphism of CYP3A5 enzyme is reported to be extremely high, which manifests with about 25 allelic variants of CYP3A5 (alleles numbered $* 1-* 9)$ ). ${ }^{13}$ Only functional CYP3A5 enzyme is known to be encoded by CYP3A $5 * 1$ allele and most common nonfunctional variant of CYP3A5 is designated as CYP3A5*3. ${ }^{11}$ GenBank sequence AC005020, has a G at position 22893 which represents the CYP3A5*3 allele and db SNP \#rs776746 is assigned for CYP3A5*3. On websitefor CYP allele nomenclature $\mathrm{CYP} 3 \mathrm{~A} 5 * 3$ is designated as $6986 \mathrm{~A}>\mathrm{G}(\mathrm{CYP} 3 \mathrm{~A} 5 * 1$ has an $\mathrm{A}$ at this position). The change of nucleotide $A$ to $G$ at this position manifests as a cryptic splice site in intron 3 which results in altered mRNA splicing. This isoform has an insertion from intron 3 , and thus, misreads the reading frame resulting in an early termination codon thereby a nonfunctional protein. ${ }^{13}$ All those who manifests with CYP3A5*3/*3 genotype are considered to be non-expressers of functional CYP3A5 enzyme.

The frequency estimate in Whites population for allele CYP3A $5 * 3$ is in the range of $0.82-0.95$ while in other ethnic groups it varies widely such as- Southwest American Indians, 0.4; Japanese, 0.85; Pacific Islanders, 0.65; African American, 0.33; Chinese, 0.65; Southeast Asians (excluding Japanese and Chinese), 0.67; and Mexicans, $0.75 .^{14}$ One of the studies statistically correlated the frequency which ranged from 0.06 in Yorubans (Nigerians) to 0.96 and the distance from the equator based on human genome diversity cell line panel (HGDP-CEPH). ${ }^{15}$

Amongst the alleles of CYP3A, polymorphisms of CYP3A5 is known to affect tacrolimus pharmacokinetics to a greater extent as compared to CYP3A4 and thus, rationale of study of these polymorphisms are presumed to be the logical approach for pharmacogenetics' application in transplants recipients. ${ }^{16}$ Path-breaking research on genetic polymorphism of CYP3A5 enzyme is credited to Macphee et al which enlightened the world in correlating the distribution of Tac for the first time. ${ }^{17}$ Individuals who are expressers of CYP3A5 genotypes (CYP3A5*1/*1 and *1/*3) are known to be metabolizing some substrates more rapidly than do CYP3A5 nonexpressers (e.g. $* 3 / * 3$ ). Individuals who are carriers of CYP3A5*1 genotype have a much higher rate of drug clearance like Tac in comparison to the other genotypes; besides, individuals with $* 1 / * 1$ genotype have a higher clearance than those who express $* 1 / * 3$ allele and further have higher clearance than $* 3 / * 3$ genotype who are also known as non-expressers. ${ }^{18}$

Guidelines for monitoring of Tac administration to patients is based on trough concentrations of transplant recipients. Also, some researchers have compared pharmacogenetic based dosing of Tac in comparison to with the standard regimen where it has been demonstrated that optimal target concentration of the Tac in recipients in shorter time interval. ${ }^{19}$ Yet another way of Tac dosing by use of mathematical equation has also been formulated based on the CYP3A5 genotype for use in adult kidney transplant recipients however the validation of same is awaited for prospective usage. ${ }^{18}$ In future, on successful validation, estimated values of clearance rate for Tac may be put to use by clinicians to determine the dose to achieve the desired trough level.

\section{METHODS}

The study was approved from institutional ethical committee and was performed in conformation of laws and regulations.

\section{Patients}

116 patients of either gender, within the age range of 2080 years were enrolled in the study, who reported 
between March 2016 till December 2016 at the nephrology center.

\section{Inclusion criteria}

Patients included in the study were those who had given written and informed consent after been explained the study in the language they understood and were willing to participate. Patients included were those who were on Tac which could be alone or with co-prescribed drugs like steroids or proton pump inhibitors but not with any other drug which could influence the enzyme CYP3A5 under study. The patients were specifically instructed to continue taking medicines at the same time every day empty stomach.

\section{Study specimen}

Whole blood samples in EDTA vacutainers were collected from vein in forearm of participant patients taking all aseptic measures. Samples which could not be processed promptly were stored at $-70^{\circ} \mathrm{C}$ till further processing.

\section{Study design}

It is a cross sectional study of Tac plasma level in whole blood sample drawn from all participants. Also, the record of daily dose of administered drug Tac was maintained from the corresponding participants. The steady-state concentration was measured once for each patient in the early post-transplant phase ranging from 2 weeks to 16 weeks after transplantation. Steady state of Tac was the dose at least 03 days after the last dose adjustment. All the patients were genotyped $(n=100)$ for the sequence variants CYP3A5*3.

\section{Analytical methods}

From each patient $10 \mathrm{ml}$ of blood sample was drawn in ethylene-diaminetetracetic acid (EDTA) vacutainer. The sample was divided into two equal parts of $5 \mathrm{ml}$ each for therapeutic drug monitoring (TDM) and genotype assay subsequently. The Tac levels were measured in $50 \mu \mathrm{l}$ whole blood sample using the DiaSorin PRO-Trac ${ }^{\mathrm{TM}}$ II tacrolimus ELISA kit on BioTek EL312e microtiter plate reader. The Tac assay was run in a microtiter plate which was precoated with goat antimouse IgG. Calibrators, controls, and samples are then added to the wells of the microtiter plate followed by addition of the antitacrolimus monoclonal antibody. After thirty minute incubation at room temperature, the tacrolimushorseradish peroxidase conjugate was added and incubated for an additional sixty minutes. The wells are then washed and chromogen was added for fifteen minute incubation. This reaction was stopped by addition of acid and the absorbance in each well was read at a dual wavelength of $450 / 630 \mathrm{~nm}$. Color development was inversely proportional to the amount of tacrolimus present in the sample. Concentrations were read from a calibrator curve.

Prevalence of the genotype CYP3A5*3 in the study population and the potential association between the CYP3A5*3 genotype and steady state dose adjusted Tac $\mathrm{C}_{0} / \mathrm{D}$ ratio $(\mu \mathrm{g} / \mathrm{l} / \mathrm{mg})$ concentration, 2 weeks to 16 weeks post transplantation was studied. Dose-adjusted concentrations of Tac was calculated by dividing the $\mathrm{C}_{0}$ by the dose in $\mathrm{mg}$ administered per day to the patients.
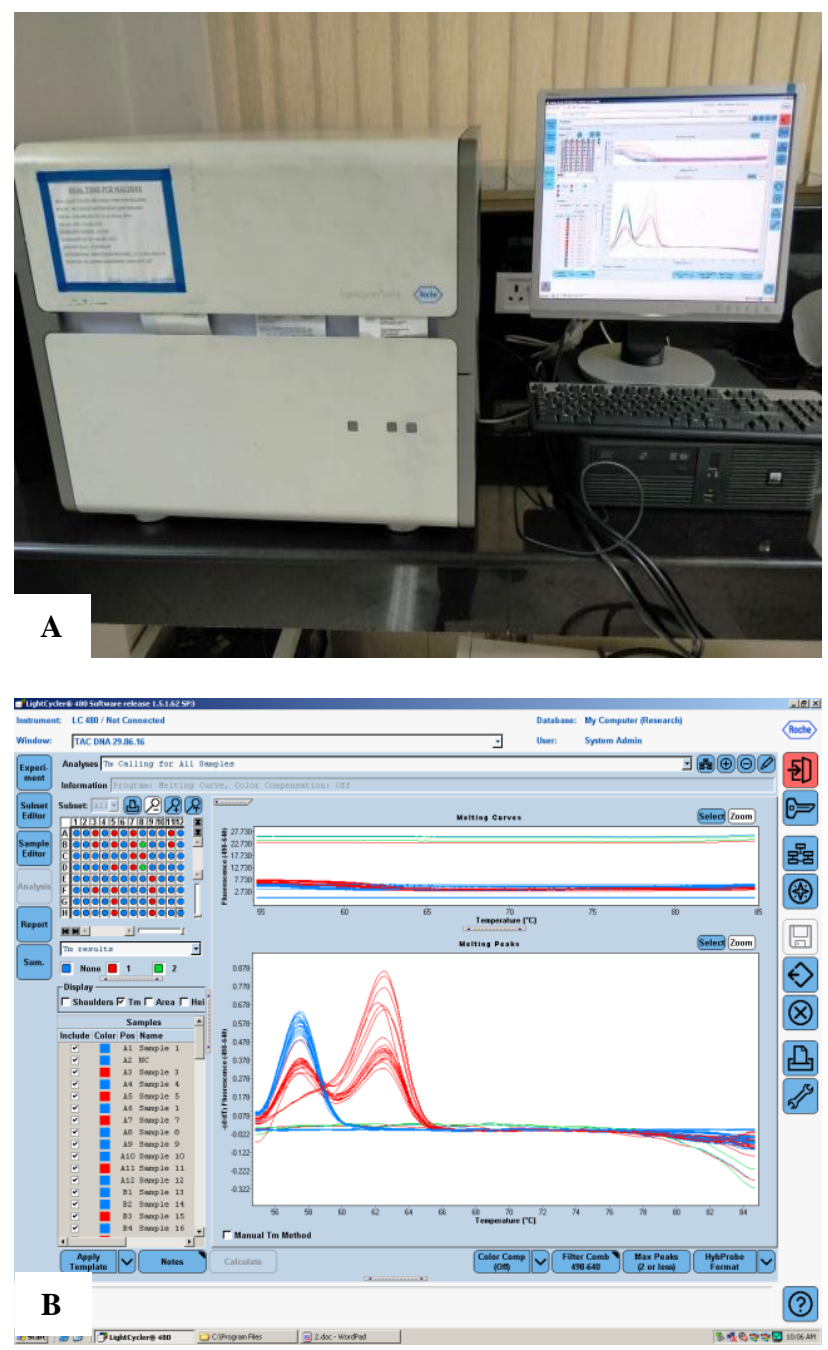

Figure 1: (A) PCR setup, (B) melting curve.

\section{Genotype analyses}

Genotyping for the CYP3A*5 (A6986G) polymorphism was performed as described by Cheung et al. 2006. The primers F16:5'-TTTgCCTCTTTgTACTTCTTCATC-3' and R16: 5'- TAgTTgTACgACACACAgCAACC-3' were used to amplify a 501 bp region from the CYP3A5 gene comprising the A6986G polymorphism in intron 3. Detection was done using the FRET probes CYP316R640: 5'-LCRed640-CCCTgTTTggACCACAT TACCCTT-3' and CYP3A5A16: 5'-FluoresceingAgCTCTTTTgTCTTTCAATATCTCT- $3^{\prime}$ which is complementary to the CYP3A*5 6986A or *1 allele. The 
sensor probe binds with a distance of one base $5^{\prime}$ to the detection probe. The PCR was carried out in a volume of $20 \mu \mathrm{l}$ with $4 \times$ light cycler genotyping DNA mastermix (Roche Diagnostics, Switzerland), $0.60 \mu \mathrm{mol} / \mathrm{l}$ of each primer, and $0.20 \mu \mathrm{mol} / 1$ of both anchor and sensor probe (Eurofins, Germany) and $5 \mu \mathrm{l}$ of genomic DNA. The PCR was carried out on a real time thermal cycler (Light Cycler 480 II, ROCHE, Diagnostics, Switzerland) with cycling parameters set as: denaturation for $10 \mathrm{~min}$ at $95^{\circ} \mathrm{C} ; 45$ cycles of $95^{\circ} \mathrm{C}$ for $10 \mathrm{~s} ; 55^{\circ} \mathrm{C}$ for $10 \mathrm{~s}$; and $72^{\circ} \mathrm{C}$ for $20 \mathrm{~s}$. Post amplification melt curve analysis was carried out with cooling to $45^{\circ} \mathrm{C}$, a hold at $45^{\circ} \mathrm{C}$ for 2 minutes and then heating slowly to $75^{\circ} \mathrm{C}$ at a rate of $0.1^{\circ} \mathrm{C} / \mathrm{s}$ with 5 acquisitions per second (Figure $1 \mathrm{~A}$ and B).

\section{Statistical analyses}

Statistical analyses were performed using SPSS software (version 20, IBM SPSS Statistics, Chicago, IL, USA). The impact of CYP3A5*3 alleles on dose-adjusted Tac concentrations was investigated by a univariate analysis of variance (ANOVA). P values less than 0.05 were considered to be statistically significant.

\section{RESULTS}

Out of 116 patients enrolled in the study, at last, data from 100 patients on Tac was analyzed with an average duration of $98 \pm 8$ days after transplantation as few participants withdrew their consent or were not traceable. Patient's demographic profile is summarized in Table 1 and also explained graphically in Figure 2 and 3 . Attributes like age, gender, weight, height, body mass index (BMI), period after transplantation, serum bilirubin, diabetes mellitus (DM) which did not significantly correlate with Tac $\mathrm{C}_{0} / \mathrm{D}$ ratio, and thus were not included in the multivariate analyses. Majority of the patients were spread in the range of 21-80 years of which the ones on Tac were maximally concentrated were in age range between 61-70 years (Figure 2). Further, there was no significant difference in genders of the patients as they were nearly equal distributed (Figure 3 ).

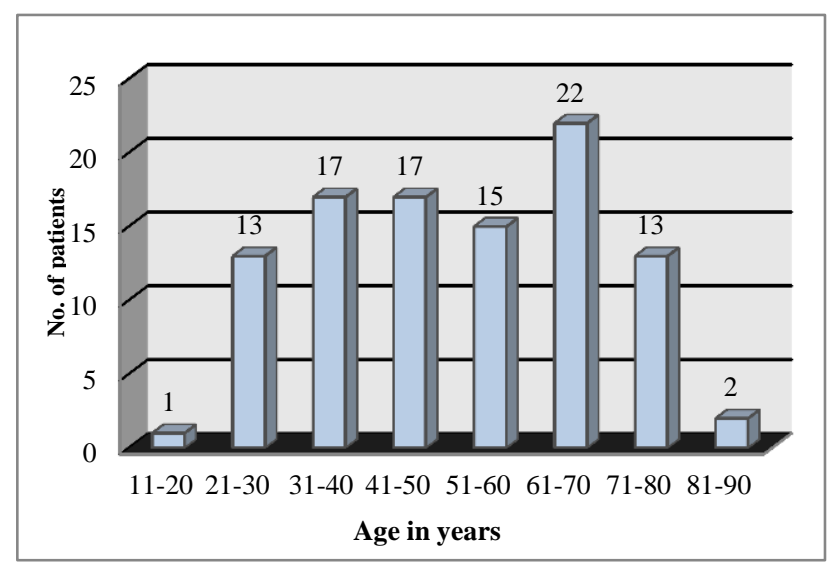

Figure 2: Age group wise distribution of subjects.
Table 1: Baseline demographic profile of subjects on Tac, median (range).

\begin{tabular}{|ll|}
\hline Demographics & Patients $(\mathbf{n}=100)$ \\
\hline Gender (male/female) & $51 / 49$ \\
\hline Age (yrs, median range) & $52(19-89)$ \\
\hline Height (m, median range) & $1.595(1.25-2.0)$ \\
\hline Weight (kg, median range) & $66(46-97)$ \\
\hline BMI (kg/m², median range) & $25.91(15.02-43.16)$ \\
\hline Diabetes mellitus & 26 \\
\hline Bilirubin (micmol/l) & $5.6(1.89-11.47)$ \\
\hline On statins & $0 / 100$ \\
\hline Steroids & $100 / 100$ \\
\hline Proton pump inhibitors & $100 / 100$ \\
\hline Dihydropyridines (n) & $56 / 100$ \\
\hline Tac dose (mg/day, range) & $1.5(0.5-8.0)$ \\
\hline $\begin{array}{l}\text { Blood concentration TAC } \\
\text { (micg/l) }\end{array}$ & $6.98(3.3-12.0)$ \\
\hline C/D ratio (micg/l/mg) & $5.0(0.63-9.20)$ \\
\hline $\begin{array}{l}\text { Time after transplantation } \\
\text { (days, range) }\end{array}$ & $64(14-112)$ \\
\hline
\end{tabular}

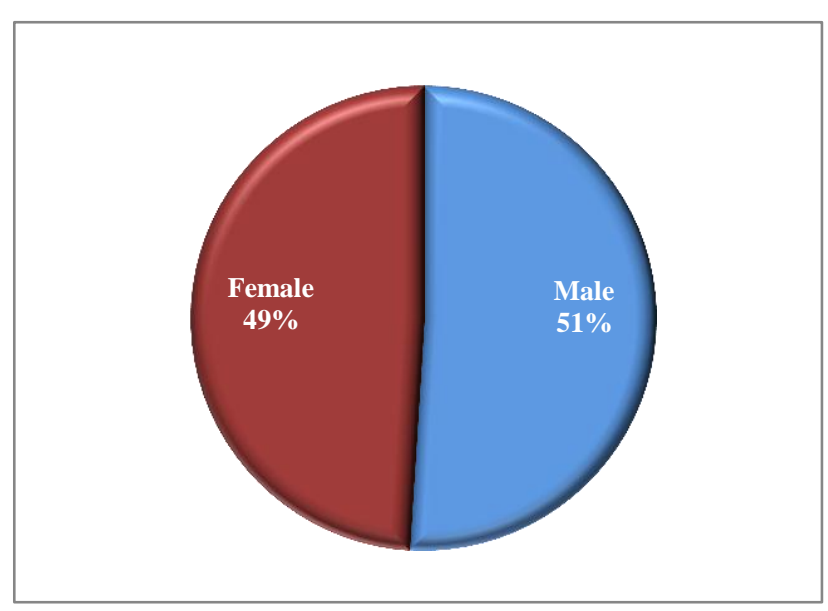

Figure 3: Gender distribution of subjects.

\section{Gene allele frequencies}

Genotype and allele frequencies of the CYP $3 A$ A $* 3$ variants are presented in Table 2. None of the genotype frequencies deviated from the Hardy-Weinberg distribution, $\mathrm{p}>0.84$, chi-squared test.

\section{Impact of genotypes on tacrolimus $C_{0} / D$ ratio}

Recipients having heterozygous CYP3A5*1 genotype manifested $70 \%$ lower mean $\mathrm{C}_{0} / \mathrm{D}$ ratio $(1.67 \pm 0.43$ $\mu \mathrm{g} / \mathrm{l} / \mathrm{mg}$ ) in comparison to homozygote carriers of CYP3A5*3 (5.60 $\pm 1.94 \mu \mathrm{g} / \mathrm{l} / \mathrm{mg}) ; \mathrm{p}<0.001$ (Figure 4).

The box-and-whisker plots indicate interquartile ranges (boxes), medians (horizontal lines in the boxes), and the highest and lowest values (whisker above and below the boxes). 
Table 2: Genotype and allele frequencies in the study population $(n=100)$ compared with allele frequencies reported in the literature.

\begin{tabular}{|llllll|} 
& \multicolumn{2}{l}{$\begin{array}{l}\text { Genotype frequencies, } \mathbf{n} \\
\text { AA }\end{array}$} & Aa & aa & Allele frequencies \\
$(\%)$ & $\begin{array}{l}\text { Reported allele frequencies in } \\
\text { Caucasians }^{\mathbf{b}}(\%)\end{array}$ \\
\hline CYP3A5 $^{*} \mathbf{3}^{\mathbf{a}}$ & 84 & 14 & 2 & 91 & $81-96$ \\
\hline
\end{tabular}

AA homozygote carriers of the major allele, Aa heterozygote carriers, aa homozygote carriers of the minor (variant) allele

${ }^{\mathrm{a}}$ Variant allele is predominant in Caucasians; ${ }^{\mathrm{b}} \mathrm{http}: / / \mathrm{www}$. ncbi.nlm.nih.gov/variation/tools/1000genomes/.

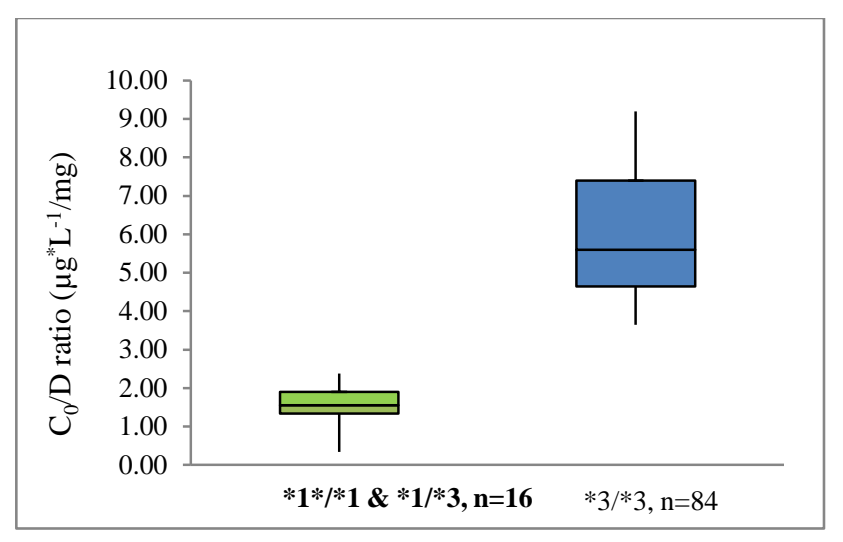

Figure 4: Tac $C_{0} / \mathrm{D}$ ratio $(\mu \mathrm{g} / \mathrm{l} / \mathrm{mg})$ CYP3A5*3.

\section{DISCUSSION}

The study revealed that Indian sub population that participated in this study was having genetic profile similar to Caucasians population for the metabolizing enzyme CYP3A5 as $91 \%$ lacked functional CYP3A5 enzyme. The allele CYP3A5*3/*3 results in alternate splicing of mRNA due to single nucleotide variant (SNV) (c.219-237A>G;rs776746) and thereby results in a truncated and ineffective metabolizing protein. Like Caucasians, in many different populations in the world, CYP3A5*3 variant is the predominant allele to the tune of approximately $80 \%$ and in our study also it was detected to be $91 \%$, in accordance with other studies. ${ }^{20}$ The lack of functional CYP3A5*3 favours patient who can be stabilized on lower Tac doses.

Few studies have also reported effect of nuclear receptor peroxisome proliferator activated receptor alpha (PPARalpha) and cytochrome $\mathrm{P} 450$ oxidoreductase (POR) on the tacrolimus $\mathrm{C}_{0} / \mathrm{D}$. Conflicting reports of effect of PPARalpha on the tacrolimus $\mathrm{C}_{0} / \mathrm{D}$ have been reported as Lunde et al have reported that variants like PPAR-alpha; c.209$1003 \mathrm{G}>\mathrm{A})$ contribute to intra and inter individual variability in CYP3A expression and activity whereas, no effect of same was reported in the studies by Kurzawski et al and Bruckmueller et al. ${ }^{19-22}$

Yet another system cytochrome P450 oxidoreductase (POR) is also implicated to alter CYP3A activity. It is a kind of flavoprotein involved in microsomal electron transfer which is intricate element of a many CYP enzymes. ${ }^{23}$ In humans, POR is highly polymorphic having most common sequence variant, $\mathrm{POR} * 28 \quad($ c. $1508 \mathrm{C}>\mathrm{T}$; rs1057868). ${ }^{24}$ It induces substitution of an amino acid (p.Ala503Val) and thereby influences the functioning of POR by altering electron binding moiety. Studies have shown that CYP3A5 expressers who also carry one or two POR*28 alleles have resulted in higher Tac dose requirements as compared with CYP3A5 expressers without POR $* 28 .{ }^{25}$ Some studies found no effect of the POR genotype variants on $T_{a c} C_{0} / D$, in contrast to Lunde et al., who reported that patients with $\mathrm{POR} * 28$ variant alleles showed $16.9 \%$ lower Tac $\mathrm{C}_{0} / \mathrm{D} .{ }^{26}$

The present study concludes that heterozygous CYP3A $5 * 1$ genotype manifests with considerable lower mean $\mathrm{C}_{0} / \mathrm{D}$ ratio in comparison to homozygote carriers of CYP3A5*3 and thus it is worthwhile to do a genetic profiling of recipients planned to be put on Tac to enhance the confidence of treating surgeons and achieve therapeutic concentration of Tac in desired range and thus achieve a higher success rate of graft survival as well as minimise the chance of rejection too.

\section{CONCLUSION}

Tacrolimus, a macrolide lactone, of fungal origin which is a strong immunosuppressive drug that has a great inter and intra plasma drug concentration variations and thus requires a stringent therapeutic drug estimation to ensure optimal plasma drug level in the recipients. Amongst various modifiable factors that have been implicated are co-administered drugs as the commonest ones which can induce or inhibit the metabolizing enzymes. Amongst the most important non-modifiable factors is the metabolizing enzyme CYP3A has been strongly implicated in Tac plasma drug levels variation besides others like Cytochrome P450 oxidoreductase and Cytochrome PPAR-alpha. This study findings further confirms that Central Indian population closely mimics Caucasian population in terms of genotype expression for CYP3A5*3, wherein they are mostly expressing a nonfunctional enzyme responsible for Tac metabolism. Also, our study further confirms that population genotype CYP3A5*1 is significantly associated with lower $\mathrm{C}_{0} / \mathrm{D}$ ratio for Tac than the ones expressing homozygous CYP3A5*3 genotype in recipients for kidney transplant. Thus, hereby it is clearly established the importance of pre-transplant genotyping, as it can allow better individualization of Tac doses pre \& post-transplant in kidney recipients.

Further, it is suggested to take up a population pharmacokinetic modelling study to suggest initial 
starting dosage of Tac based on profiling of CYP3 genetics which is likely to have considerable impact in optimization of drug dosing for improving the transplant acceptance without a chance of failure or undue intolerance of the immunosuppressant drug under study.

Funding: No funding sources

Conflict of interest: None declared

Ethical approval: The study was approved by the Institutional Ethics Committee

\section{REFERENCES}

1. Kino T, Hatanaka H, Hashimoto M, Nishiyama M, Goto T, Okuhara $\mathrm{M}$, et al. FK506, a novel immunosuppressant isolated from a Streptomyces I. Fermentation, isolation, and physio-chemical and biological characteristics. J Antibiot (Tokyo). 1987;40(9):1249-55.

2. Wallemacq PE, Reding R. FK506 (tacrolimus), a novel immunosuppressant in organ transplantation: clinical, biomedical, and analytical aspects. Clin Chemis. 1993;39:2219-28.

3. Scott LJ, McKeage K, Keam SJ, Plosker GL. Tacrolimus: a further update of its use in the management of organ transplantation. Drugs. 2003;63(12):1247-97.

4. Venkataramanan R, Swaminathan A, Prasad T, Jain A, Zuckerman S, Warty $\mathrm{V}$, et al. Clinical pharmacokinetics of tacrolimus. Clin Pharmacokinet. 1995;29:404-30.

5. Staatz CE, Tett SE. Clinical pharmacokinetics and pharmacodynamics of tacrolimus in solid organ transplantation. Clin Pharmacokinet. 2004;43(10):623-53.

6. Wallemacq P, Armstrong VW, Brunet M, Haufroid V, Holt DW, Johnston A, et al. Opportunities to optimize tacrolimus therapy in solid organ transplantation: report of the European consensus conference. Ther Drug Monit. 2009;31(2):139-52.

7. Barraclough KA, Isbel NM, Kirkpatrick CM, Lee KJ, Taylor PJ, Johnson DW, et al. Evaluation of limited sampling methods for estimation of tacrolimus exposure in adult kidney transplant recipients. $\mathrm{Br} \mathrm{J}$ Clin Pharmacol. 2011;71(2):207-23.

8. Katsakiori PF, Papapetrou EP, Sakellaropoulos GC, Goumenos DS, Nikiforidis GC, Christodoulos, et al. Factors affecting the long-term response to tacrolimus in renal transplant patients: Pharmacokinetic Pharmacogenetic approach. Int J Med Sci. 2010;7(2):94-100.

9. deJonge H, Kuypers DR. Pharmacogenetics in solid organ transplantation:current status and future directions. Transplant Rev. (Orlando). 2008;22(1):620.

10. Jusko WJ, Thomson AW, Fung J, McMaster P, Wong SH, Zylber-Katz E, et al. Consensus document:Therapeutic monitoring of tacrolimus (FK506). Ther Drug Monit. 1995;17(6):606-14.
11. Lamba J, Hebert JM, Schuetz EG, Kleinb TE, Altman RB. Pharm GKB summary: very important pharmacogene information for CYP3A5. Pharmacogenet Genomics. 2012;22(7):555-8.

12. Lamba JK, Lin YS, Schuetz EG, Thummel KE. Genetic contribution to variable human CYP3Amediated metabolism. Adv Drug Deliv Rev. 2002;54(10):1271-94.

13. Kuehl P, Zhang J, Lin Y, Lamba J, Assem M, Schuetz J, et al. Sequence diversity in CYP3A promoters and characterization of the genetic basis of polymorphic CYP3A5 expression. Nat Genet. 2001;27:383-91.

14. Lee SJ, Usmani KA, Chanas B, Ghanayem B, Xi T, Hodgson E, et al. Genetic findings and functional studies of human CYP3A5 single nucleotide polymorphisms in different ethnic groups. Pharmacogenetics. 2003;13:461-472.

15. Thompson EE, Kuttab-Boulos H, Witonsky D, Yang L, Roe BA, Di Rienzo A. CYP3A variation and the evolution of salt-sensitivity variants. Am J Hum Genet. 2004;75:1059-69.

16. Evans WE. Pharmacogenomics: marshalling the human genome to individualize drug therapy. Evans WE. Pharmacogenomics: marshalling the human genome to individualise drug therapy. Gut. 2003;52(2):10-8.

17. MacPhee IA, Holt DW. A pharmacogenetic strategy for immunosuppression based on the CYP3A5 genotype. Transplantation. 2008;85:163-5.

18. Passey C, Birnbaum AK, Brundage RC, Oetting WS, Israni AK, Jacobson PA. Dosing equation for tacrolimus using genetic variants and clinical factors. Br J Clin Pharmacol. 2011;72:948-57.

19. Lunde I, Bremer S, Midtvedt K, Mohebi B, Dahl M, Bergan S, et al. The influence of CYP3A, PPARA, and POR genetic variants on the pharmacokinetics of tacrolimus and cyclosporine in renal transplant recipients. Eur J Clin Pharmacol. 2014;70(6):685-93.

20. Thervet E, Loriot MA, Barbier S, Buchler M, Ficheux M, Choukroun G, et al. Optimization of initial tacrolimus dose using pharmacogenetic testing. Clin Pharmacol Ther. 2010;87:721-6.

21. Kurzawski M, Malinowski D, Dziewanwski K, Drozdzik M. Pharmacogenet. Genomics. 2014;24(8):397-400.

22. Bruckmueller H1, Werk AN, Renders L, Feldkamp T, Tepel M, Borst C, et al. Drug Monit. 2015;37(3):288-95.

23. Roy JN, Lajoie J, Zijenah LS, Barama A, Poirier C, Ward BJ, et al. CYP3A5 genetic polymorphisms in different ethnic populations. Drug Metab Dispos. 2005;33(7):884-7.

24. Wang D, Guo Y, Wrighton SA, Cooke GE, Sadee W. Intronic polymorphism in CYP3A4 affects hepatic expression and response to statin drugs. Pharmacogenomics J. 2011;11(4):274-86.

25. Klein K, Thomas M, Winter S, Nussler AK, Niemi M, Schwab M, et al. PPARA: a novel genetic 
determinant of CYP3A4 in vitro and in vivo. Clin Pharmacol Ther. 2012;91(6):1044-52.

26. Elens L, Hesselink DA, Bouamar R, Budde K, de Fijter JW, De Meyer M, et al. Impact of POR*28 on the pharmacokinetics of tacrolimus and cyclosporine A in renal transplant patients. Ther Drug Monit. 2014;36(1):71-9.
Cite this article as: Gupta AK, Ramashankar MM, Kumar M. Genetic variation of CYP3A and its influence on the pharmacokinetics of tacrolimus, a calcineurin inhibitor. Int J Basic Clin Pharmacol 2019;8:2039-45. 